\title{
DRG-Handbuch 2008 - \\ Zahl der Fallpauschalen steigt erneut Vorsicht vor Überraschungen im Kodieralltag
}

Auch wenn das deutsche Fallpauschalensystem (G-DRG) inzwischen den Kinderschuhen entwachsen scheint und sich inhaltlich und strukturell immer stärker von seinem Vorbild, dem australischen DRG-System entfernt - abgeschlossen ist seine Entwicklung auch im Jahr 2008 noch nicht. Obwohl gerade komplexe Behandlungsgeschehen im neuen DRG-Katalog besser abgebildet und dadurch einträglicher vergütet werden, sind es vor allem teuere Behandlungen, die Intensivmedizin oder Mehrfacheingriffe, die wohl noch weiteren Verbesserungen bedürfen.

Insgesamt scheinen die meisten Kliniken mit dem leistungsorientierten Vergütungssystem relativ zufrieden, auch wenn die Umstellung mit einem erheblichen Aufwand verbunden gewesen ist, den insbesondere das ärztliche Personal der Krankenhäuser durch die zunehmenden Kodieraufgaben zu spüren bekam. Seit den ersten Gehversuchen des G-DRG-Systems („german diagnosis related groups“) hat sich viel getan, abgeschlossen ist die Entwicklung sicherlich noch nicht.

Immer mehr Fallpauschalen - wo bleibt da die Übersichtlichkeit? Zwar fällt der Unterschied zum Vorjahr nicht mehr allzu drastisch aus, die Zahl der Fallpauschalen ist aber auch im Jahr 2008 weiter gestiegen. Insgesamt zählt das System jetzt 1137 DRGs - das sind genau 55 mehr als noch im letzten Jahr. Darüber hinaus können die Kliniken jetzt 115 Zusatzentgelte abrechnen, auch das sind zehn mehr als noch im Jahr 2007.

Dr. Frank Heimig, der Geschäftsführer des Instituts für das Entgeltsystem im Krankenhaus (InEK) ist zuversichtlich, dass die Klassifikation im Großen und Ganzen fertig sei. Bei den wenigen Pauschalen, die jetzt noch fehlen, geht es seiner Meinung nach um die fachgerechte Vergütung für Spezialleistungen, die nur von wenigen Kliniken überhaupt erbracht werden, also um den Bereich der Spitzen und Maximalversorgungsmedizin, sagte der InEK-Chef gegenüber der Ärzte-Zeitung.

Wenn auch nicht mehr viele neue DRGFallpauschalen zu erwarten sind, das sich entwickelnde System ist schon jetzt äußerst komplex. Ein Problem sieht Heimig darin aber nicht, denn $90 \%$ der Kliniken arbeiten mit weniger als 700 Pauschalen. Deutlicher wird das Bild noch, wenn man nur die DRGs berücksichtigt, die mehr als einmal pro Krankenhaus kodiert wurden. Dann fallen im überwiegenden Teil der Krankenhäuser nur noch 250-400 Fallpauschalen an.

Dr. Andreas Priefler, Pressesprecher der Deutschen Krankenhausgesellschaft, hält den neuen DRG-Katalog daher für eine tragfähige Lösung. Er ist der Meinung, „dass mit dem neuen Katalog das komplexe Behandlungsgeschehen noch genauer abgebildet und dadurch adäquater vergütet werden kann“, wie er gegenüber der Ärzte-Zeitung hervorhob.

Die viel beschworenen „blutigen Entlassungen" habe es jedoch nicht gegeben, auch wenn die Krankenhausverweildauer im Zeitverlauf immer weiter reduziert werden konnte. War der Mittelwert der Verweildauer im Jahr 2004 noch 7,94 Tage, sank dieser im Laufe von zwei Jahren auf 7,58 Tage - absolut gesehen sicherlich kein großer Unterschied, in Prozentzahlen ausgedrückt aber immerhin eine Differenz von 4,5\%.

\section{Matrix hat sich}

\section{entscheidend verändert}

Entscheidend verändert gegenüber den Vorjahren hat sich im Jahr 2008 die DRG-Matrix. So fungieren beispielsweise Nebendiagnosen wie Harnwegsinfekte oder Hypokaliämien, die in den Vorjahren besonders häufig kodiert wurden, nicht mehr als Kostentrenner und sind konsequenterweise aus der Matrix eli- miniert oder zumindest abgewertet worden. Eher seltene Codes wie die Transplantatabstoßung oder das Lockedin-Syndrom haben sich dagegen einen Platz erobert und wurden aufgewertet. „Krankenhäuser, die in den letzen Jahren vornehmlich danach kodierten, ob eine Diagnose Geld einbringt oder nicht, erleben nun unter Umständen böse Überraschungen bei ihrer Leistungsplanung“, schreibt Dr. Michael Wilke, München, im letzten Trillium-Report (Ausgabe 5 (2), Seite 104), „da die neuen erlössteigernden Kodes in der Vergangenheit nicht oder selten kodiert wurden und so der Case-Mix absinkt. Es lohnt also weiterhin, auch Diagnosen zu kodieren, die aktuell nicht direkt zum Case-Mix beitragen.“

\section{Schweregradbemessung erhält immer mehr Relevanz}

Nicht gerade förderlich für die Transparenz sei die vor einigen Jahren begonnene Schweregradbemessung, so Wilke weiter, die eine immer höhere Relevanz erhalte. In Einzelfällen könne dies dazu führen, dass die Kodierung erschwerender Diagnosen und Prozeduren nicht zu der erhofften höheren Vergütung führe, sondern sogar Mindererlöse nach sich ziehe.

In den aktuellen DRGs gilt dies nicht mehr für den klassischen Fall der „endoskopischen oder diagnostischen Eingriffe bei Stoffwechselerkrankungen ohne Komorbiditäten oder Komplikationen (CC)“ (K40Z), der seit Jahren Verluste einbrachte, wenn zur Blasenspiegelung Nebendiagnosen kodiert wurden. Dafür habe sich zum Beispiel die Erlösminderung für Herzinfarktpatienten (F43C) mit HIV-Infektion gegenüber 2007 verdoppelt, so Wilke.

sts

Quelle: Abschlussbericht zur Weiterentwicklung des G-DRG-Systems für das Jahr 2008 Klassifikation, Katalog und Bewertungsrelationen, verfasst vom Institut für das Entgeltsystem im Krankenhaus $\mathrm{GmbH}$, Siegburg 